\title{
Some characterizations of error bound for non-lower semicontinuous functions
}

\author{
Miantao Chao ${ }^{1,2,3}$, Xiuping Wang ${ }^{4}$ and Dongying Liang ${ }^{5 *}$
}

\author{
"Correspondence: \\ liangdy_go@126.com \\ ${ }^{5}$ Guangxi Vocational and Technical \\ College of Communications, \\ Nanning, P.R. China \\ Full list of author information is \\ available at the end of the article
}

\begin{abstract}
In this paper, we study the error bound of non-lower semicontinuous functions. First, we extend the concepts of strong slope and global slope to the non-lower semicontinuous functions. Second, by using the two concepts, some characterizations of the existence of the global and local error bounds are given for the non-lower semicontinuous functions. Especially, we get a necessary and sufficient condition of global error bounds for the non-lower semicontinuous functions. Moreover, it is shown by an example that the strong slope and the global slope cannot characterize the error bounds of the non-lower semicontinuous functions. Third, we emphasize the special case of convex functions defined on Euclidean space. Although the strong slope and the global slope cannot characterize the error bounds of the non-lower semicontinuous functions, they could be used to characterize the error bounds of the non-lower semicontinuous convex functions. We get several necessary and sufficient conditions of global error bounds for the non-lower semicontinuous convex functions.
\end{abstract}

Keywords: Non-lower semicontinuous function; Error bound; Convex function; Strong slop; Global slop

\section{Introduction}

Error bounds have important applications in the sensitivity analysis of mathematical programming and in the convergence analysis of some algorithms. For example, the theory of error bound can be useful in the convergence analysis in the algorithm in solving optimization problem [1-3], variational inequality problem [4, 5], and identifying the active constraint [6]. Many researchers have focused their attention on the study of the error bound (see [7-25] and the references therein). We refer the interested reader to the surveys by Azé [8], Fabian et al. [13], Lewis and Pang [18], and Pang [23].

Although there is an extensive literature body on error bound, there is little literature on the error bound of the non-lower semicontinuous functions. In this paper, we study error bounds for the non-lower semicontinuous functions. The study of an inequality defined by a non-lower semicontinuous function arose from a broad class of outer approximation methods for convex optimization (see [26] and the references therein). It is also a theoretical interest to study error bounds without lower semicontinuity [16]. Some commonly studied non-lower semicontinuous functions include the indicator functions of non-closed sets. For example, the feasible direction cones of a closed convex set may not be closed, thus their indicator functions may not be lower semicontinuous [27].

(c) The Author(s) 2019. This article is distributed under the terms of the Creative Commons Attribution 4.0 International License (http://creativecommons.org/licenses/by/4.0/), which permits unrestricted use, distribution, and reproduction in any medium, provided you give appropriate credit to the original author(s) and the source, provide a link to the Creative Commons license, and indicate if changes were made. 
The paper is organized as follows. In Sect. 2, we provide some preliminaries. In Sect. 3, we extend the concepts of strong slope and global slope to the non-lower semicontinuous functions. By using the two concepts, some characterizations of the global and local error bounds are given for the non-lower semicontinuous functions. Especially, we get a necessary and sufficient condition of global error bounds for the non-lower semicontinuous functions. In Sect. 4, we emphasize the special case of the non-lower semicontinuous convex functions defined on Euclidean space. We get several necessary and sufficient conditions of global error bounds for the non-lower semicontinuous convex functions. Moreover, the results imply that the strong slope and the global slope could be used to characterize the error bound of the non-lower semicontinuous convex functions. Finally, we make some conclusions in Sect. 5 .

\section{Preliminaries}

Throughout the paper, unless otherwise specified, let $(X, d)$ be a complete metric space, $f: X \rightarrow R \cup\{+\infty\}$ be a non-lower semicontinuous function. The lower semicontinuous hull of the function $f$ is defined by $\operatorname{cl} f(x):=\min \left\{\liminf _{y \rightarrow x} f(y), f(x)\right\}$. As usual, $\operatorname{dom} f:=$ $\{x \in X \mid f(x)<+\infty\}$ denotes the effective domain of $f, f_{+}(x):=\max \{f(x), 0\}$. Let $[f \leq 0]:=$ $\{x \in X \mid f(x) \leq 0\},[f=0]:=\{x \in X \mid f(x)=0\}$, and $[f>0]:=\{x \in X \mid f(x)>0\}$. For $U \subseteq X$ and $\rho \in(0,+\infty)$, we define

$$
B_{\rho}(U):=\{x \in X \mid d(x, U)<\rho\} \quad \text { and } \quad \bar{B}_{\rho}(U):=\{x \in X \mid d(x, U) \leq \rho\} \text {, }
$$

where $d(x, U):=\inf \{d(x, y) \mid y \in U\}$ with the convention that $d(x, \emptyset)=+\infty$. Let cl $U$ denote the closure of the set $U$.

First, we recall the notation of global and local error bound.

Definition 2.1 We say that $f$ has a global error bound if there exists a positive real number $\sigma$ such that

$$
\sigma d(x,[f \leq 0]) \leq f(x) \quad \text { for all } x \in X \text {. }
$$

Let $\sigma(f)$ denote the supremum of $\tau \in[0,+\infty)$ such that

$$
\tau d(x,[f \leq 0]) \leq f(x) \quad \text { for all } x \in X .
$$

Definition 2.2 Let $\bar{x} \in X$ such that $f(\bar{x}) \leq 0$. We say that $f$ has a local error bound at $\bar{x}$ if there exist a neighborhood $U$ of $\bar{x}$ and a positive constant $\sigma$ such that

$$
\sigma d(x,[f \leq 0]) \leq f_{+}(x) \quad \text { for all } x \in U
$$

Next, we recall the notion of strong slope introduced by De Giorgi et al. [28]. The strong slope was used by several authors to give characterizations of error bound for the lower semicontinuous functions (see $[7,9,29]$ ). 
Definition 2.3 The strong slope of function $f$ at $x \in \operatorname{dom} f$ is defined by

$$
|\nabla f|(x)=\limsup _{y \rightarrow x} \frac{(f(x)-f(y))_{+}}{d(x, y)} .
$$

For $x \notin \operatorname{dom} f$, let $|\nabla f|(x)=+\infty$.

The following notion (called nonlocal slope in [30]) was first introduced by Ngai and Théra in [21, Theorem 2.1].

Definition 2.4 The global slope of function $f$ at $x \in \operatorname{dom} f$ is defined by

$$
{ }^{\diamond}|\nabla f|(x)=\sup _{y} \frac{\left(f(x)-f_{+}(y)\right)_{+}}{d(x, y)} .
$$

For $x \notin \operatorname{dom} f$, let ${ }^{\diamond}|\nabla f|(x)=+\infty$.

In Sect. 3, we will give an example to show that the strong slope and the global slope cannot be used to characterize the error bound of the non-lower semicontinuous functions (see Example 3.1).

\section{Global and local error bounds}

In this section, we extend the concepts of strong slope and global slope to the non-lower semicontinuous functions and use them to give some necessary and/or sufficient conditions for the global and local error bounds of the non-lower semicontinuous functions.

Definition 3.1 The closed strong slope of $f$ at $x \in \operatorname{dom} f$ is defined by

$$
|\nabla f|^{*}(x)=\limsup _{u \rightarrow x} \frac{(\mathrm{cl} f(x)-f(u))_{+}}{d(x, u)} .
$$

For $x \notin \operatorname{dom} f$, let $|\nabla f|^{*}(x)=+\infty$.

Definition 3.2 The closed global slope of $f$ at $x$ is defined by

$$
{ }^{\diamond}|\nabla f|^{*}(x)=\sup _{u} \frac{\left(\mathrm{cl} f(x)-f_{+}(u)\right)_{+}}{d(x, u)} .
$$

For $x \notin \operatorname{dom} f$, let ${ }^{\diamond}|\nabla f|^{*}(x)=+\infty$.

According to the above definitions, one can easily get the following proposition.

\section{Proposition 3.1}

(i) If $x \in X \backslash \operatorname{cl}[f \leq 0]$, then ${ }^{\diamond}|\nabla f|(x) \geq|\nabla f|(x)$ and ${ }^{\diamond}|\nabla f|^{*}(x) \geq|\nabla f|^{*}(x)$.

(ii) $\diamond|\nabla f|^{*}(x) \leq \diamond|\nabla f|(x)$ and $|\nabla f|^{*}(x) \leq|\nabla f|(x)$.

(iii) If $f$ is lower semicontinuous, then

$$
{ }^{\diamond}|\nabla f|^{*}(x)={ }^{\diamond}|\nabla f|(x) \text { and }|\nabla f|^{*}(x)=|\nabla f|(x) \text {. }
$$


The following proposition implies that the closed strong slope and the closed global slope of the function $f$ is the strong slope and the global slope of the function $\mathrm{cl} f$, respectively.

\section{Proposition 3.2}

(i) $|\nabla f|^{*}(x)=\lim \sup _{u \rightarrow x} \frac{(\mathrm{cl} f(x)-\mathrm{cl} f(u))_{+}}{d(x, u)}=|\nabla \mathrm{cl} f|(x)$,

(ii) $\diamond|\nabla f|^{*}(x)=\sup _{u} \frac{\left(\mathrm{cl} f(x)-(\mathrm{cl} f)_{+}(u)\right)_{+}}{d(x, u)}={ }^{\diamond}|\nabla \mathrm{cl} f|(x)$.

Proof We only prove (ii), and one can get (i) in a similar way. (ii) If $\operatorname{cl} f(x)=+\infty$, then the conclusion is clearly established. Without loss of generality, suppose that $\mathrm{cl} f(x)<$ $+\infty$. Since $(\operatorname{cl} f)_{+}(u) \leq f_{+}(u),{ }^{\diamond}|\nabla f|^{*}(x) \leq{ }^{\diamond}|\nabla \operatorname{cl} f|(x)$. Next, we prove that ${ }^{\diamond}|\nabla f|^{*}(x) \geq$ $\diamond|\nabla \mathrm{cl} f|(x)$.

If ${ }^{\diamond}|\nabla \mathrm{cl} f|^{*}(x)=0$, then $\operatorname{cl} f(x) \leq(\mathrm{cl} f)_{+}(u) \leq f_{+}(u)$ for all $u \in X \backslash\{x\}$. From the definition of ${ }^{\diamond}|\nabla f|^{*}(x)$, one has ${ }^{\diamond}|\nabla f|^{*}(x)=0$.

Next, we assume that ${ }^{\diamond}|\nabla \mathrm{cl} f|^{*}(x)>0$. Furthermore, $\operatorname{cl} f(x)>0$. Let the sequence $\left\{u_{n}\right\}$ be such that ${ }^{\diamond}|\nabla \mathrm{cl} f|^{*}(x)=\lim _{n \rightarrow \infty} \frac{\left(\mathrm{cl} f(x)-(\mathrm{cl} f)_{+}\left(u_{n}\right)\right)_{+}}{d\left(x, u_{n}\right)}$. Since ${ }^{\diamond}|\nabla \mathrm{cl} f|^{*}(x)>0$ and $\mathrm{cl} f(x)<$ $+\infty$, the sequence $\left\{u_{n}\right\}$ is bounded. There exists a subsequence $\left\{u_{n_{k}}\right\}$ of $\left\{u_{n}\right\}$ such that $\lim _{n_{k} \rightarrow \infty} u_{n_{k}}=x^{*}$. We consider two cases.

$1^{\circ} x^{*}=x$. From the definition of the closure hull, one has $\lim _{n_{k} \rightarrow \infty} \operatorname{cl} f\left(u_{n_{k}}\right) \geq \mathrm{cl} f(x)$. Thus, cl $f\left(u_{n_{k}}\right)>0$ for large enough $n_{k}$ and ${ }^{\diamond}|\nabla \mathrm{cl} f|(x)=\lim _{n_{k} \rightarrow \infty} \frac{\left(\operatorname{cl} f(x)-(\mathrm{cl} f)\left(u_{n_{k}}\right)\right)_{+}}{d\left(x, u_{n_{k}}\right)}$. For every $u_{n_{k}}$, take a point $v_{k}$ such that

$$
f\left(v_{k}\right)>0,\left|f\left(v_{k}\right)-\operatorname{cl} f\left(u_{n_{k}}\right)\right|<\frac{1}{k} d\left(x, u_{n_{k}}\right), d\left(x, v_{k}\right) \geq \frac{k-1}{k} d\left(x, u_{n_{k}}\right) .
$$

Thus,

$$
\begin{aligned}
{ }^{\diamond}|\nabla f|^{*}(x) & \geq \lim _{k \rightarrow \infty} \frac{\left(\mathrm{cl} f(x)-f\left(v_{k}\right)\right)_{+}}{d\left(x, v_{k}\right)} \\
& \geq \lim _{n_{k} \rightarrow \infty} \frac{\left(\mathrm{cl} f(x)-(\mathrm{cl} f)\left(u_{n_{k}}\right)-\frac{1}{k} d\left(x, u_{n_{k}}\right)\right)_{+}}{\frac{k-1}{k} d\left(x, u_{n_{k}}\right)} \\
& ={ }^{\diamond}|\nabla \operatorname{cl} f|(x) .
\end{aligned}
$$

$2^{\circ} x^{*} \neq x$. In this case, $d\left(x, x^{*}\right)>0$. Since $\operatorname{cl} f\left(x^{*}\right) \leq \liminf _{n_{k} \rightarrow \infty} \operatorname{cl} f\left(u_{n_{k}}\right), \diamond|\nabla \operatorname{cl} f|(x)=$ $\frac{\left(\mathrm{cl} f(x)-(\mathrm{cl} f)_{+}\left(x^{*}\right)\right)_{+}}{d\left(x, x^{*}\right)}$.

Let the sequence $\left\{v_{k}\right\}$ be such that $d\left(x^{*}, v_{k}\right) \leq \frac{1}{k}$ and $\left|f\left(v_{k}\right)-\operatorname{cl} f\left(x^{*}\right)\right|<\frac{1}{k}$. Thus

$$
\begin{aligned}
{ }^{\diamond}|\nabla f|^{*}(x) & \geq \liminf _{k \rightarrow \infty} \frac{\left(\mathrm{cl} f(x)-f_{+}\left(v_{k}\right)\right)_{+}}{d\left(x, v_{k}\right)} \\
& \geq \liminf _{k \rightarrow \infty} \frac{\left(\mathrm{cl} f(x)-\mathrm{cl} f_{+}\left(x^{*}\right)-\frac{1}{k}\right)_{+}}{d\left(x, v_{k}\right)} \\
& =\liminf _{k \rightarrow \infty} \frac{\left(\mathrm{cl} f(x)-\operatorname{cl} f_{+}\left(x^{*}\right)-\frac{1}{k}\right)_{+}}{d\left(x, x^{*}\right)} \frac{d\left(x, x^{*}\right)}{d\left(x, v_{k}\right)} \\
& ={ }^{\diamond}|\nabla \mathrm{cl} f|(x) .
\end{aligned}
$$

The following proposition gives some sufficient conditions for the nonemptiness of the set $[f \leq 0]$. 
Proposition 3.3 If $\inf _{X \backslash \mathrm{cl}[f \leq 0]}{ }^{\diamond}|\nabla f|^{*}(x)>0$ or $\inf _{X \backslash \mathrm{cl}[f \leq 0]}|\nabla f|^{*}(x)>0$, then
(i) $[f \leq 0] \neq \emptyset$,
(ii) $\quad \operatorname{cl}[f \leq 0]=[\mathrm{cl} f \leq 0]$.

Proof Since $|\nabla f|^{*}(x) \leq^{\ominus}|\nabla f|^{*}(x)$ for every $x \in X \backslash \operatorname{cl}[f \leq 0]$, we only need to prove that the conclusions are true under the condition $\inf _{X \backslash \mathrm{cl}[f \leq 0]}{ }^{\diamond}|\nabla f|^{*}(x)>0$.

(i) Let $\sigma:=\inf _{X \backslash \mathrm{cl}[f \leq 0]} \diamond|\nabla f|^{*}(x)$. Suppose for contradiction that $[f \leq 0]=\emptyset$. Thus $\inf _{X} \operatorname{cl} f(x) \geq 0$. Let $\bar{x} \in \operatorname{dom} f$, then there exist $\sigma^{\prime} \in(0, \sigma)$ and $r \in(0,+\infty)$ such that

$$
\operatorname{cl} f(\bar{x}) \leq \inf _{X} \operatorname{cl} f(x)+\sigma^{\prime} r
$$

By virtue of the Ekeland variational principle [31], there exists $x \in \bar{B}_{r}(\bar{x})$ with $\mathrm{cl} f(x) \leq$ $\operatorname{cl} f(\bar{x})$ such that $\operatorname{cl} f(x)<\operatorname{cl} f(y)+\sigma^{\prime} d(x, y) \leq f(y)+\sigma^{\prime} d(x, y)$ for every $y \in X \backslash\{x\}$. Thus ${ }^{\diamond}|\nabla f|^{*}(x) \leq \sigma^{\prime}<\sigma$, contradicting the assumption.

(ii) $\operatorname{cl}[f \leq 0] \subseteq[\mathrm{cl} f \leq 0]$ is obvious. Next, we prove that $\operatorname{cl}[f \leq 0] \supseteq[\mathrm{cl} f \leq 0]$.

If $x \notin \operatorname{cl}[f \leq 0]$, then

$$
{ }^{\diamond}|\nabla f|^{*}(x)=\sup _{u} \frac{\left(\mathrm{cl} f(x)-f_{+}(u)\right)_{+}}{d(x, u)}>0 .
$$

Thus $\mathrm{cl} f(x)>0$. This implies that $x \notin[\mathrm{cl} f \leq 0]$.

The following theorem gives two global error bound criteria for the non-lower semicontinuous functions.

\section{Theorem 3.1}

(i) If $\inf _{X \backslash \mathrm{cl}[f \leq 0]}|\nabla f|^{*}(x) \geq \sigma$, then $f_{+}(x) \geq \sigma d(x,[f \leq 0]), \forall x \in X$.

(ii) $\inf _{X \backslash \mathrm{cl}[f \leq 0]}{ }^{\diamond}|\nabla f|^{*}(x) \geq \sigma$ if and only if $f_{+}(x) \geq \sigma d(x,[f \leq 0]), \forall x \in X$.

Proof If $\sigma \leq 0$, then the conclusions are clearly established. In the following, we assume that $\sigma>0$. From Proposition 3.3, one can get

$$
[f \leq 0] \not \emptyset \quad \text { and } \quad \operatorname{cl}[f \leq 0]=[\mathrm{cl} f \leq 0] .
$$

From Proposition 3.2, we have

$$
\inf _{X \backslash \mathrm{cl} f \leq 0]}|\nabla \operatorname{cl} f|^{*}(x) \geq \sigma, \quad \inf _{X \backslash \mathrm{cl}[f \leq 0]} \diamond|\nabla \operatorname{cl} f|^{*}(x) \geq \sigma .
$$

By [9, Theorem 2.1], [21, Theorem 2.1], and [16, Theorem 2.1], Theorem 3.1 holds.

The following example implies that the strong slope and the global slope cannot be used to characterize the error bound of the non-lower semicontinuous functions.

Example 3.1 Let $Q$ denote the set of all rational numbers and $P$ denote the set of all irrational number. Let $f: R \rightarrow R$ be defined as

$$
f(x)= \begin{cases}-1, & \text { if } x \leq 0, \\ 1, & \text { if } x \in Q \cap(0,+\infty), \\ 1-x+[x], & \text { if } x \in P \cap(0,+\infty),\end{cases}
$$


where $[x]$ denotes the largest integer not less than $x$. It is easy to see that $x \backslash \operatorname{cl}[f \leq 0]=[f>$ $0]$. The function $f$ is non-lower semicontinuous and $\inf _{[f>0]}{ }^{\diamond}|\nabla f|^{*}(x)=\inf _{[f>0]}|\nabla f|^{*}(x)=0$. It follows from Theorem 3.1(ii) that $f$ does not have a global error bound. One can easily get $\inf _{[f>0]} \diamond|\nabla f|(x)=\inf _{[f>0]}|\nabla f|(x)=1>0$. This implies that the strong slope and the global slope cannot be used to characterize the error bound of the non-lower semicontinuous functions.

Next, we give the characterizations of local error bounds for the non-lower semicontinuous functions.

Theorem 3.2 Let $U \subseteq[f \leq 0], C:=X \backslash \mathrm{cl}[f \leq 0], \rho>0$; and let $A:=\bar{B}_{\rho}(U) \cap C$ and $D:=$ $B_{2 \rho}(U) \cap C$. If $\operatorname{cl}[f \leq 0]=[\mathrm{cl} f \leq 0]$, then

$$
\inf _{A} \frac{f(x)}{d(x,[f \leq \alpha])} \geq \inf _{D}^{\diamond}|\nabla f|^{*}(x) \geq \inf _{D}|\nabla f|^{*}(x) .
$$

Proof By Proposition 3.1(i), we only need to show the first inequality. We may assume that the left-hand side of the inequality is finite. Thus $A \neq \emptyset$, and so $[f \leq 0] \neq \emptyset$. For any $\sigma>\inf _{A} \frac{f(x)}{d(x,[f \leq 0])}$, there exists $\bar{x} \in A$ such that

$$
f(\bar{x})<\sigma d(\bar{x},[f \leq 0])
$$

Let $r \in(0, d(\bar{x},[f \leq 0]))$ such that $f(\bar{x})<\sigma r$. Let $g(x):=f_{+}(x)$. We have

$$
\operatorname{cl} g(\bar{x})<\inf _{X} \operatorname{cl} g(x)+\sigma r .
$$

By virtue of the Ekeland variational principle [31], there exists $\hat{x} \in \bar{B}_{r}(\bar{x})$ such that $\operatorname{cl} g(\hat{x}) \leq$ $\operatorname{cl} g(\bar{x})$ and $\operatorname{cl} g(\hat{x})<\operatorname{cl} g(y)+\sigma d(\hat{x}, y)$ for every $y \in X \backslash\{\hat{x}\}$. Note that $r<d(\bar{x},[f \leq 0]) \leq$ $d(\bar{x}, U) \leq \rho$. For $x \in \bar{B}_{r}(\bar{x}) \subset B_{2 \rho}(U)$, we have $\operatorname{cl} f(x)>0$ since $\operatorname{cl}[f \leq 0]=[\mathrm{cl} f \leq 0]$. Thus $\hat{x} \in D$. Furthermore,

$$
0<\operatorname{cl} f(\hat{x})<f_{+}(y)+\sigma d(\hat{x}, y), \quad \forall y \in X \backslash\{\hat{x}\}
$$

One can get ${ }^{\diamond}|\nabla f|^{*}(\hat{x}) \leq \sigma$. Thus $\inf _{D}{ }^{\diamond}|\nabla f|^{*}(x) \leq \sigma$, and the conclusion follows.

As a special case of Theorem 3.2 that $U$ is singleton, some characterizations of the local error bounds are obtained as follows.

Corollary 3.1 Let $C:=X \backslash \operatorname{cl}[f \leq 0], \bar{x} \in[f \leq 0], \rho>0$, and $D:=B_{2 \rho}(\bar{x}) \cap C$. Assume that $\operatorname{cl}[f \leq 0]=[\operatorname{cl} f \leq 0]$. If $\inf _{D}|\nabla f|^{*}(x) \geq \sigma$ or $\inf _{D}{ }^{\diamond}|\nabla f|_{\alpha}^{*}(x) \geq \sigma$, then $f_{+}(x) \geq \sigma d(x,[f \leq \alpha])$, $\forall x \in B_{\rho}(\bar{x})$, that is, $f$ has a local error bound at $\bar{x}$.

\section{The convex case}

In this section, we focus our discussion on the convex case. We show that the strong slope and the global slope could be used to characterize the error bound of the non-lower semicontinuous convex functions under mild assumptions. Throughout this section, let $X=R^{n}$ be the $n$-dimensional Euclidean space with Euclidean norm $\|\cdot\|$ and $f: R^{n} \rightarrow R \cup\{+\infty\}$ 
be a non-lower semicontinuous convex function. Let $\operatorname{ri}(\operatorname{dom} f)$ denote the relative interior of the set $\operatorname{dom} f$. It is well known from convex analysis (see, for example, [32]) that $\operatorname{cl} f(x)=f(x)$ for all $x \in \operatorname{ri}(\operatorname{dom} f)$.

Proposition 4.1 If $x \in X \backslash \mathrm{cl}[f \leq 0]$, then

$$
{ }^{\diamond}|\nabla f|(x)=|\nabla f|(x) \text { and }{ }^{\diamond}|\nabla f|^{*}(x)=|\nabla f|^{*}(x) \text {. }
$$

Proof According to Proposition 3.1, we only need to show that ${ }^{\diamond}|\nabla f|^{*}(x) \leq|\nabla f|^{*}(x)$ and ${ }^{\diamond}|\nabla f|(x) \leq|\nabla f|(x)$ for all $x \in X \backslash \operatorname{cl}[f \leq 0]$. We only show that ${ }^{\diamond}|\nabla f|^{*}(x) \leq|\nabla f|^{*}(x)$ for all $x \in X \backslash \operatorname{cl}[f \leq 0]$. Similarly, one can show that ${ }^{\diamond}|\nabla f|(x) \leq|\nabla f|(x)$ for all $x \in X \backslash \operatorname{cl}[f \leq 0]$.

Let $x \in X \backslash \operatorname{cl}[f>0]$. If ${ }^{\diamond}|\nabla f|^{*}(x)=0$, then $|\nabla f|^{*}(x)=0$. Next, we assume that ${ }^{\diamond}|\nabla f|^{*}(x)>$ 0 . Let $\lambda \in(0,1)$. For any $y(\neq x)$ such that $f(y) \leq \operatorname{cl} f(x)$, one has

$$
\begin{aligned}
\frac{\left(\mathrm{cl} f(x)-f_{+}(y)\right)_{+}}{\|x-y\|} & \leq \frac{(\mathrm{cl} f(x)-\mathrm{cl} f(y))_{+}}{\|x-y\|} \\
& \leq \frac{(\operatorname{cl} f(x)-\operatorname{cl} f(\lambda x+(1-\lambda) y))_{+}}{\|x-(\lambda x+(1-\lambda) y)\|} \\
& \leq \lim _{\lambda \uparrow 1} \frac{(\operatorname{cl} f(x)-\operatorname{cl} f(\lambda x+(1-\lambda) y))_{+}}{\|x-(\lambda x+(1-\lambda) y)\|} .
\end{aligned}
$$

Let $\left\{z_{\lambda}\right\}_{\lambda \in(0,1)}$ be such that

$$
f\left(z_{\lambda}\right)=\operatorname{cl} f(\lambda x+(1-\lambda) y)+o\left(\left\|x-z_{\lambda}\right\|\right) \quad \text { as } \lambda \uparrow 1,
$$

and

$$
\left\|x-z_{\lambda}\right\|=\|x-(\lambda x+(1-\lambda) y)\|+\circ\left(\left\|x-z_{\lambda}\right\|\right) \quad \text { as } \lambda \uparrow 1 .
$$

Thus

$$
\begin{aligned}
\frac{\left(\mathrm{cl} f(x)-f_{+}(y)\right)_{+}}{\|x-y\|} & \leq \lim _{\lambda \uparrow 1} \frac{\left(\mathrm{cl} f(x)-f\left(z_{\lambda}\right)+o\left(\left\|x-z_{\lambda}\right\|\right)\right)_{+}}{\left\|x-z_{\lambda}\right\|+o\left(\left\|x-z_{\lambda}\right\|\right)} \\
& =\lim _{\lambda \uparrow 1} \frac{\left(\mathrm{cl} f(x)-f\left(z_{\lambda}\right)\right)_{+}}{\left\|x-z_{\lambda}\right\|} \leq \limsup _{z \rightarrow x} \frac{(\mathrm{cl} f(x)-f(z))_{+}}{\|x-z\|}=|\nabla f|^{*}(x) .
\end{aligned}
$$

Thus

$$
\diamond|\nabla f|^{*}(x)=\sup _{y \neq x} \frac{\left(\mathrm{cl} f(x)-f_{+}(y)\right)_{+}}{\|x-y\|} \leq|\nabla f|^{*}(x) .
$$

The proof is completed.

Lemma 4.1 If $[f \leq 0] \neq \emptyset, \eta:=\inf _{\mathrm{cl}(\operatorname{dom} f) \backslash \mathrm{ri}(\operatorname{dom} f)} \operatorname{cl} f(x)>0$, and $\beta \in(0, \eta)$, then

$$
\inf _{[f=\beta]}{ }^{\circ}|\nabla f|^{*}(x) \leq \inf _{[f>\beta]} \diamond|\nabla f|^{*}(x)
$$


Proof Assume that $\inf _{[f=\beta]}{ }^{\diamond}|\nabla f|^{*}(x)>0$ and $\inf _{[f>\beta]}{ }^{\diamond}|\nabla f|^{*}(x)<+\infty$, thus $\beta>\inf _{X} f$. For any fixed $\sigma \in\left(\inf _{[f>\beta]^{\diamond}}|\nabla f|^{*}(x),+\infty\right)$. Let $\bar{x} \in[f>\beta]$ and $\sigma>^{\diamond}|\nabla f|^{*}(\bar{x})$. Let

$$
g(y):=f(y)+\sigma\|y-\bar{x}\|+\delta(y \mid[f \leq \beta])
$$

where $\delta(y \mid[f \leq \beta])$ is the indicator function of the set $[f \leq \beta]$.

We claim that $g(y)>\operatorname{cl} f(\bar{x})$ for all $y \neq \bar{x}$. Suppose for a contradiction that there exists $\hat{y} \in$ $[f \leq \beta]$ such that $f(\hat{y})+\sigma\|\hat{y}-\bar{x}\|+\delta(\hat{y} \mid[f \leq \beta]) \leq \operatorname{cl} f(\bar{x})$. Since $\beta \in(0, \eta)$, then $\hat{y} \in \operatorname{ri}(\operatorname{dom} f)$. By [32, Theorem 6.1], one has $\lambda \hat{y}+(1-\lambda) \bar{x} \in \operatorname{ri}(\operatorname{dom} f)$. By [32, Theorem 10.1], one has $\operatorname{cl} f(\hat{y})=f(\hat{y})$ and $\operatorname{cl} f(\lambda \hat{y}+(1-\lambda) \bar{x})=f(\lambda \hat{y}+(1-\lambda) \bar{x})$ for all $\lambda \in(0,1)$. Since $\liminf _{\lambda \downarrow 0} f(\lambda \hat{y}+$ $(1-\lambda) \bar{x}) \geq \operatorname{cl} f(\bar{x})$, then $f(\lambda \hat{y}+(1-\lambda) \bar{x})>0$ for sufficiently small $\lambda \in(0,1)$. If $\lambda \in(0,1)$ is sufficiently small, then

$$
\begin{aligned}
{ }^{\diamond}|\nabla f|^{*}(\bar{x}) & \geq \frac{\left(\mathrm{cl} f(\bar{x})-f_{+}(\lambda \hat{y}+(1-\lambda) \bar{x})\right)_{+}}{\|\bar{x}-(\lambda \hat{y}+(1-\lambda) \bar{x})\|} \\
& =\frac{(\operatorname{cl} f(\bar{x})-f(\lambda \hat{y}+(1-\lambda) \bar{x}))_{+}}{\| \bar{x}-(\lambda \hat{y}+(1-\lambda) \bar{x})) \|} \geq \frac{(\operatorname{cl} f(\bar{x})-f(\hat{y}))_{+}}{\| \bar{x}-\hat{y}) \|} \geq \sigma
\end{aligned}
$$

a contradiction, where the second inequality follows from $f(\lambda \hat{y}+(1-\lambda) \bar{x})=\operatorname{cl} f(\lambda \hat{y}+(1-$ $\lambda) \bar{x}) \leq \lambda \operatorname{cl} f(\bar{x})+(1-\lambda) \operatorname{cl} f(\hat{y})$ and $\operatorname{cl} f(\hat{y})=f(\hat{y})$.

Let $\varepsilon \in\left(0, \sigma-\diamond|\nabla f|^{*}(\bar{x})\right)$ and $\bar{y} \in[f \leq \beta]$. Since $g(y)>\operatorname{cl} f(\bar{x})$ for all $y \neq \bar{x}$, there exists $\bar{r}_{\varepsilon}>0$ such that

$$
\operatorname{cl} g(\bar{y}) \leq \inf \operatorname{cl} g(y)+\varepsilon \bar{r}_{\varepsilon} .
$$

By virtue of the Ekeland variational principle [31], there exists $y_{\varepsilon}$ such that

$$
\operatorname{cl} g(y)+\varepsilon\left\|y-y_{\varepsilon}\right\|>\operatorname{cl} g\left(y_{\varepsilon}\right) \quad \text { for all } y \neq y_{\varepsilon} .
$$

Thus $y_{\varepsilon} \in[f \leq \beta] \subseteq \operatorname{ri}(\operatorname{dom} f)$ and

$$
f(y)+\sigma\|y-\bar{x}\| \geq f\left(y_{\varepsilon}\right)+\sigma\left\|y_{\varepsilon}-\bar{x}\right\|-\varepsilon\left\|y-y_{\varepsilon}\right\| \quad \text { for all } y \in[f \leq \beta] .
$$

We claim that $f\left(y_{\varepsilon}\right)=\beta$. Indeed, we may assume, for contradiction, that $f\left(y_{\varepsilon}\right)<\beta$. Let $z_{\varepsilon}$ be a point in the open segment $\left(\bar{x}, y_{\varepsilon}\right)$ with $f\left(z_{\varepsilon}\right)=\beta>0$. Then $z_{\varepsilon} \in \operatorname{ri}(\operatorname{dom} f)$. Writing (1) for $y:=z_{\varepsilon}$ yields

$$
f\left(z_{\varepsilon}\right)-f\left(y_{\varepsilon}\right) \geq \sigma\left(\left\|y_{\varepsilon}-\bar{x}\right\|-\left\|z_{\varepsilon}-\bar{x}\right\|\right)-\varepsilon\left\|z_{\varepsilon}-y_{\varepsilon}\right\|=(\sigma-\varepsilon)\left\|z_{\varepsilon}-y_{\varepsilon}\right\|,
$$

where the equality follows from $z_{\varepsilon} \in\left(\bar{x}, y_{\varepsilon}\right)$. For $\lambda \in(0,1)$ is sufficiently small, one has

$$
\begin{aligned}
{ }^{\diamond}|\nabla f|^{*}(\bar{x}) & \geq \frac{\left(\mathrm{cl} f(\bar{x})-f_{+}\left(z_{\varepsilon}\right)\right)_{+}}{\left\|\bar{x}-z_{\varepsilon}\right\|} \\
& \geq \frac{\left(\mathrm{cl} f\left(z_{\varepsilon}\right)-f_{+}\left((1-\lambda) z_{\varepsilon}+\lambda y_{\varepsilon}\right)\right)_{+}}{\left\|z_{\varepsilon}-\left((1-\lambda) z_{\varepsilon}+\lambda y_{\varepsilon}\right)\right\|} \\
& =\frac{\left(\operatorname{cl} f\left(z_{\varepsilon}\right)-f\left((1-\lambda) z_{\varepsilon}+\lambda y_{\varepsilon}\right)\right)_{+}}{\left\|z_{\varepsilon}-\left((1-\lambda) z_{\varepsilon}+\lambda y_{\varepsilon}\right)\right\|}
\end{aligned}
$$




$$
\geq \frac{\left(f\left(z_{\varepsilon}\right)-f\left(y_{\varepsilon}\right)\right)_{+}}{\left\|z_{\varepsilon}-y_{\varepsilon}\right\|} \geq \sigma-\varepsilon
$$

a contradiction. Thus $f\left(y_{\varepsilon}\right)=\beta$.

Now, we derive from (1) again that

$$
f\left(y_{\varepsilon}\right)-f(y) \leq \sigma\left(\|y-\bar{x}\|-\left\|y_{\varepsilon}-\bar{x}\right\|\right)+\varepsilon\left\|y-y_{\varepsilon}\right\| \leq(\sigma+\varepsilon)\left\|y-y_{\varepsilon}\right\| \quad \text { for all } y \in[f \leq \beta] .
$$

Thus

$$
{ }^{\diamond}|\nabla f|^{*}\left(y_{\varepsilon}\right)=\sup _{y} \frac{\left(f\left(y_{\varepsilon}\right)-f_{+}(y)\right)_{+}}{\left\|y_{\varepsilon}-y\right\|} \leq \sigma+\varepsilon,
$$

which shows that $\inf _{[f=\beta]}{ }^{\diamond}|\nabla f|^{*}(\bar{x}) \leq \sigma$. Thus $\inf _{[f=\beta]}^{\diamond}|\nabla f|^{*}(\bar{x}) \leq \inf _{[f>\beta]}{ }^{\diamond}|\nabla f|^{*}(x)$.

Proposition 4.2 If $[f \leq 0] \neq \emptyset$ and $\inf _{\mathrm{cl}(\operatorname{dom} f) \backslash \mathrm{ri}(\operatorname{dom} f)} \mathrm{cl} f(x)>0$, then

$$
\begin{aligned}
\inf _{X \backslash \mathrm{cl}[f \leq 0]}|\nabla f|(x) & =\inf _{X \backslash \mathrm{cl}[f \leq 0]} \diamond|\nabla f|(x) \\
& =\inf _{X \backslash \mathrm{cl}[f \leq 0]} \diamond|\nabla f|^{*}(x)=\inf _{X \backslash \mathrm{cl} l f \leq 0]}|\nabla f|^{*}(x) .
\end{aligned}
$$

Proof By Proposition 4.1, we only need to prove

$$
\inf _{X \backslash \mathrm{cl}[f \leq 0]}{ }^{\diamond}|\nabla f|(x)=\inf _{X \backslash \mathrm{cl}[f \leq 0]}{ }^{\diamond}|\nabla f|^{*}(x) .
$$

By Lemma 4.1, we have

$$
\begin{aligned}
\inf _{[f>\beta]}^{\diamond}|\nabla f|(x) & \geq \inf _{[f>\beta]} \diamond|\nabla f|^{*}(x) \geq \inf _{[f=\beta]}^{\diamond}|\nabla f|^{*}(x) \\
& \geq \inf _{[\leq \leq \beta] \backslash \mathrm{cl}[f \leq 0]} \diamond|\nabla f|^{*}(x)=\inf _{[\leq \leq \beta] \backslash \mathrm{cl}[f \leq 0]} \diamond|\nabla f|(x),
\end{aligned}
$$

where the equality follows from $[f \leq \beta] \subseteq \operatorname{ri}(\operatorname{dom} f)$. Thus

$$
\begin{aligned}
\inf _{X \backslash \mathrm{cl}[f \leq 0]} \diamond|\nabla f|(x) & =\min \left\{\inf _{[f \leq \beta] \backslash \mathrm{cl}[f \leq 0]} \diamond|\nabla f|(x), \inf _{[f>\beta] \backslash \mathrm{cl}[f \leq 0]} \diamond|\nabla f|(x)\right\} \\
& =\inf _{[f \leq \beta] \backslash \mathrm{cl}[f \leq 0]} \diamond|\nabla f|(x),
\end{aligned}
$$

and

$$
\begin{aligned}
\inf _{X \backslash \mathrm{cl} f \leq 0]} \diamond|\nabla f|^{*}(x) & =\min \left\{\inf _{[f \leq \beta \backslash \mathrm{cl}[f \leq 0]} \diamond|\nabla f|^{*}(x), \inf _{[f>\beta] \backslash \mathrm{cl} f \leq 0]} \diamond|\nabla f|^{*}(x)\right\} \\
& =\inf _{[f \leq \beta] \backslash \mathrm{cl}[f \leq 0]}|\nabla f|^{*}(x) .
\end{aligned}
$$

The above three formulas imply that

$$
\inf _{X \backslash \mathrm{cl} l f \leq 0]}{ }^{\diamond}|\nabla f|(x)=\inf _{X \backslash \mathrm{cl} l f \leq 0]}{ }^{\diamond}|\nabla f|^{*}(x) .
$$

By Theorem 3.1 and Proposition 4.2, we have the following result, which gives characterizations of global error bounds for the non-lower semicontinuous convex functions. 


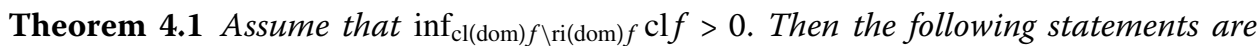
equivalent:

(i) $f_{+}(x) \geq \sigma d(x,[f \leq 0]), \forall x \in X$

(ii) $\inf _{X \backslash \mathrm{cl}[f \leq 0]}{ }^{\diamond}|\nabla f|(x) \geq \sigma$.

(iii) $\inf _{X \backslash \mathrm{cl} f \leq 0]}|\nabla f|(x) \geq \sigma$.

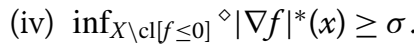

(v) $\inf _{X \backslash \mathrm{cl}[f \leq 0]}|\nabla f|^{*}(x) \geq \sigma$.

\section{Conclusions}

In this paper, we establish some necessary and/or sufficient conditions of global and local error bounds for the non-lower semicontinuous functions. We also emphasize the special case of convex functions defined on Euclidean space. We get several necessary and sufficient conditions of global error bounds for convex functions defined on Euclidean space.

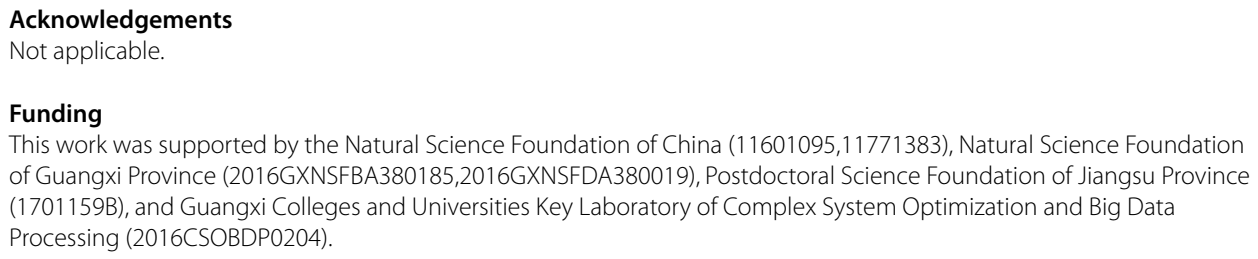
of Guangxi Province (2016GXNSFBA380185,2016GXNSFDA380019), Postdoctoral Science Foundation of Jiangsu Province (1701159B), and Guangxi Colleges and Universities Key Laboratory of Complex System Optimization and Big Data Processing (2016CSOBDP0204).

\section{Availability of data and materials}

Not applicable.

\section{Competing interests}

The authors declare that they have no competing interests.

\section{Authors' contributions}

MTC carried out the idea of this paper. XPW and DYL helped to draft the manuscript. All authors read and approved the final manuscript.

\section{Authors' information}

M.T. Chao, Ph.D., associate professor; X.P. Wang, lecturer; D.Y. Liang, associate professor.

\section{Author details}

'School of Mathematical Sciences, Nanjing Normal University, Nanjing, P.R. China. ${ }^{2}$ Guangxi Colleges and Universities Key Laboratory of Complex System Optimization and Big Data Processing, Yulin Normal University, Yulin, P.R. China. ${ }^{3}$ College of Mathematics and Information Science, Guangxi University, Nanning, P.R. China. ${ }^{4}$ School of Pharmacy, Liaocheng University, Liaocheng, P.R. China. ${ }^{5}$ Guangxi Vocational and Technical College of Communications, Nanning, P.R. China.

\section{Publisher's Note}

Springer Nature remains neutral with regard to jurisdictional claims in published maps and institutional affiliations.

Received: 7 November 2018 Accepted: 28 January 2019 Published online: 12 February 2019

\section{References}

1. Hong, M.Y., Luo, Z.Q.: On the linear convergence of the alternating direction method of multipliers. Math. Program. $162,165-199(2017)$

2. Luo, Z.Q.: New error bounds and their applications to convergence analysis of iterative algorithms. Math. Program. $88,341-355(2000)$

3. Zhang, $\mathrm{H}$.: The restricted strong convexity revisited: analysis of equivalence to error bound and quadratic growth. Optim. Lett. 11, 811-833 (2017)

4. Li, G.Y., Ng, K.F.: Error bounds of generalized D-gap functions for nonsmooth and nonmonotone variational inequality problems. SIAM J. Optim. 20, 667-690 (2009)

5. Luo, Z.Q.. Pang, J.S.: Error bounds for analytic systems and their applications. Math. Program. 67, 1-28 (1994)

6. Facchinei, F., Fischer, A., Kanzow, C.: On the accurate identification of active constraints. SIAM J. Optim. 9, 14-32 (1999)

7. Azé, D., Corvellec, J.N.: On the sensitivity analysis of Hoffman constants for systems of linear inequalities. SIAM J. Optim. 12, 913-927 (2001)

8. Azé, D.: A survey on error bounds for lower semicontinuous functions. ESAIM Proc. 13, 1-17 (2003) 
9. Azé, D., Corvellec, J.N.: Characterizations of error bounds for lower semicontinuous functions on metric spaces. ESAIM Control Optim. Calc. Var. 10, 409-425 (2004)

10. Bosch, P., Jourani, A., Henrion, R.: Sufficient conditions for error bounds and applications. Appl. Math. Optim. 50, 161-181 (2004)

11. Boț, R.I., Csetnek, E.R.: Error bound results for convex inequality systems via conjugate duality. Top 20, 296-309 (2012)

12. Chao, M.T., Cheng, C.Z.: Linear and nonlinear error bounds for lower semicontinuous functions. Optim. Lett. 8 $1301-1312(2014)$

13. Fabian, M.J., Henrion, R., Kruger, A.Y., Outrata, J.V.: Error bounds: necessary and sufficient conditions. Set-Valued Anal. $18,121-149(2010)$

14. He, Y.R., Sun, J.: Second-order sufficient conditions for error bounds in Banach spaces. SIAM J. Optim. 17, 795-805 (2006)

15. Hoffman, A.J.: On approximate solutions of systems of linear inequalities. J. Res. Natl. Bur. Stand. 49, 263-265 (1952)

16. Hu, H., Wang, Q.: Local and global error bounds for proper functions. Pac. J. Optim. 6, 177-186 (2010)

17. Huang, L.R., Ng, K.F.: On first-order and second-order conditions for error bounds. SIAM J. Optim. 14, 1057-1073 (2004)

18. Lewis, A.S., Pang, J.S.: Error bounds for convex inequality systems, in generalized convexity, generalized monotonicity: recent results Marseille. Nonconvex Optim. Appl. 27, 75-110 (1998)

19. Li, G.Y.: Global error bounds for piecewise convex polynomials. J. Res. Natl. Bur. Stand. 137, 37-64 (2013)

20. Ng, K.F., Zheng, X.Y.: Error bounds for lower semicontinuous functions in normed spaces. SIAM J. Optim. 12, 1-17 (2001)

21. Ngai, H.V., Théra, M.: Error bounds in metric spaces and application to the perturbation stability of metric regularity SIAM J. Optim. 19, 1-20 (2008)

22. Ngai, H.V., Théra, M.: Error bounds for systems of lower semicontinuous functions in Asplund spaces. Math. Program. 116, 397-427 (2009)

23. Pang, J.S.: Error bounds in mathematical programming. Math. Program. 79, 299-332 (1997)

24. $\mathrm{Wu}, \mathrm{Z}$., Ye, J.J.: First-order and second-order conditions for error bounds. SIAM J. Optim. 14, 621-645 (2003)

25. Zheng, X.Y., Wei, Z.: Perturbation analysis of error bounds for quasi-subsmooth inequalities and semi-infinite constraint systems. SIAM J. Optim. 22, 41-65 (2003)

26. Combettes, P.: Strong convergence of block-iterative outer approximation methods for convex optimization. SIAM J. Control Optim. 38, 538-565 (2000)

27. Bakan, A., Deutsch, F., Li, W.: Strong CHIP, normality, and linear regularity of convex sets. Trans. Am. Math. Soc. 357, 3831-3863 (2005)

28. Giorgi, E.D., Marino, A., Tosques, M.: Problemi di spazi metrici e curve di massima pendenza (Evolution problems in metric spaces and curves of maximal slope). Atti Accad. Naz. Lincei, Rend. Cl. Sci. Fis. Mat. Nat. 68, 180-187 (1980)

29. Corvellec, J.N., Motreanu, V.V.: Nonlinear error bounds for lower semicontinuous functions on metric spaces. Math. Program. 114, 291-319 (2008)

30. Ngaic, H.V., Kruger, A.Y., Théra, M.: Slopes of multifunctions and extensions of metric regularity. Math. Program. 40 , 355-369 (2012)

31. Ekeland, I.: Nonconvex minimization problems. Bull. Am. Math. Soc. 1, 443-474 (1979)

32. Rockafellar, R.: Convex Analysis. Princeton University Press, Princeton (1970)

\section{Submit your manuscript to a SpringerOpen ${ }^{\circ}$ journal and benefit from:}

- Convenient online submission

- Rigorous peer review

- Open access: articles freely available online

- High visibility within the field

- Retaining the copyright to your article

Submit your next manuscript at $\boldsymbol{~ s p r i n g e r o p e n . c o m ~}$ 